\title{
Inferior Thyroid Artery
}

National Cancer Institute

\section{Source}

National Cancer Institute. Inferior Thyroid Artery. NCI Thesaurus. Code C53022.

One of the three branches of the thyroid axis that supplies the thyroid gland and branches to form the ascending cervical, esophageal, inferior laryngeal, muscular and tracheal arteries. 